Journal of Patient-Centered

Volume 4

Issue 4 -- Health Disparities and Inequities: Part

Article 23 I

$11-6-2017$

\title{
Quality Improvement Study for Postpartum Hypertension Readmissions
}

Molly K. Lepic

Sara M. Stanenas

Carla J. Kelly

Deborah Simpson

Jeffrey A. Stearns

Follow this and additional works at: https://aah.org/jpcrr

Part of the Health Services Administration Commons, Medical Education Commons, Obstetrics and Gynecology Commons, and the Women's Health Commons

\section{Recommended Citation}

Lepic MK, Stanenas SM, Kelly CJ, Simpson D, Stearns JA. Quality improvement study for postpartum hypertension readmissions. J Patient Cent Res Rev. 2017;4:261-2.

Published quarterly by Midwest-based health system Advocate Aurora Health and indexed in PubMed Central, the Journal of Patient-Centered Research and Reviews (JPCRR) is an open access, peer-reviewed medical journal focused on disseminating scholarly works devoted to improving patient-centered care practices, health outcomes, and the patient experience. 
Background: Prenatal care/deliveries within our family medicine clinics have declined, perhaps because patients are unware that our clinics provide these services. With lower volumes, clinicians may feel less comfortable with current skills/knowledge of obstetric (OB) care. Purpose: Increase family medicine clinic OB numbers, patient awareness, and clinician comfort/knowledge in OB.

Methods: English-facile patients (18-50 years), residents and faculty at Aurora family medicine residency clinics were included. Patients were provided preintervention surveys upon check-in. Residents/ faculty were surveyed via Survey Monkey. Changes made based on initial survey results were: 1) increasing systemwide awareness that our caregivers provide $\mathrm{OB}$ care, through fliers at emergency departments/urgent cares or posters in clinic waiting rooms; 2) keeping at least one same-day visit for $\mathrm{OB}$ patients; 3 ) distributing standard $\mathrm{OB}$ note templates to residents/faculty; and 4) placing patient educational handouts at each clinic. Patients, residents and faculty were reassessed at 9 months postintervention. Surveys were analyzed with Fisher's exact tests.

Results: Respondents to the preintervention survey included 83 patients, 26 residents and 19 faculty; 61 patients, 23 residents and 21 faculty responded to the postintervention survey. On both preand post-surveys, patients knew that their providers delivered babies ( $59 \%$ vs $57 \%$, respectively; $\mathrm{P}=0.86$ ). However, only $22 \%$ and $33 \%$ of patients, respectively, had a doctor at our clinics deliver their baby or partner's baby $(\mathrm{P}=0.25)$. Even so, $95 \%$ and $100 \%$ of patients, respectively, would recommend their friends or family to our family practice clinics if they became pregnant $(\mathrm{P}=0.14)$. On the pre-survey, $38 \%$ of residents felt clinic OB numbers were adequate versus $70 \%$ following intervention $(\mathrm{P}<0.05)$. On both pre- and postsurveys, residents planned on incorporating obstetric or prenatal care into their future practice ( $42 \%$ vs $52 \%$, respectively; $\mathrm{P}=0.57$ ). On both pre- and post-surveys, faculty felt comfortable with $\mathrm{OB}$ skills and knowledge ( $53 \%$ vs $62 \%$, respectively; $\mathrm{P}=0.75$ ). Lifestyle was the most common reason faculty gave for why they stopped doing deliveries (37\% vs 33\%, respectively).

Conclusion: Implementation of changes to our OB workflow resulted in non-statistically significant improvements in viewpoints toward OB. Resident feelings of $\mathrm{OB}$ number adequacy significantly improved following intervention. Further study in multiple clinics could confirm the effectiveness and reasons for success of our interventions.

\section{Brain Imaging in Older Patients With Delirium}

\section{Laila M. Hasan, Ariba Khan, Maharaj Singh, Michael L. Malone}

\section{Department of Geriatrics, Aurora University of Wisconsin Medical Group; Aurora Research Institute}

Background: Delirium is a common, serious and costly condition in older patients admitted to the hospital. This study describes the prevalence and results of brain imaging among a cohort of older hospitalized patients with and without delirium.

Purpose: Investigate the frequency and results of brain imaging in older patients with delirium as compared to those without delirium. Methods: This was a cross-sectional study. Data were collected on hospitalized patients age 65 years or older who were admitted to 3 hospitals in Milwaukee, Wisconsin, during a 1-month period in the fall of 2013. Subjects were tested for delirium via the "Confusion Assessment Method" by researchers for another study. The collected data included demographics, presence of delirium, computed tomography (CT) and magnetic resonance imaging (MRI), and results of the imaging procedures. The imaging studies were done as a part of their medical care. The authors reviewed the radiologist's final readings of the imaging studies. For all categorical variables, chi squared/Fisher's test was used with alpha of 0.05 .

Results: A total of 92 patients were included in the study. Prevalence of delirium was $17.4 \%$. Mean age was 77 years. Overall, $24 \%$ had a CT and $9 \%$ an MRI, with the most common abnormal finding being chronic microvascular changes (13\%). CT scan was performed in $44 \%$ of patients with delirium and $20 \%$ of patients without delirium $(\mathrm{P}=0.04)$. MRI was performed in zero patients with delirium and $11 \%$ without delirium $(\mathrm{P}=0.34)$. When patients with delirium were compared with patients without delirium, respectively: normal imaging was described in 1 vs 2 patients $(\mathrm{P}=0.70)$; cerebral atrophy in 3 vs $6(\mathrm{P}=0.99)$; chronic microvascular changes in 2 vs 10 $(\mathrm{P}=0.17)$; and acute hematoma (subdural or intraparenchymal) in 3 (43\%) vs $0(\mathrm{P}=0.02)$.

Conclusion: In this limited study, patients with delirium were noted to be more likely to have had a CT scan. Older patients with delirium had a variety of findings on brain imaging, some of which were more clinically relevant. No specific imaging changes were diagnostic for delirium.

\section{Quality Improvement Study for Postpartum Hypertension Readmissions}

Molly K. Lepic, Sara M. Stanenas, Carla J. Kelly, Deborah Simpson, Jeffrey A. Stearns

Department of Obstetrics and Gynecology, Aurora Sinai Medical Center, Aurora University of Wisconsin Medical Group; Departments of Family Medicine and Academic Affairs, Aurora Health Care

Background: Hospital readmission rates are a focus of the Centers for Medicare and Medicaid Services. This was identified as a system opportunity to improve health care quality and patient education in order to reduce preventable readmissions. In 2009, $27 \%$ of obstetric readmissions were due to hypertensive disease, and preventable readmissions regarding hypertension are flagged as an area for quality improvement in our health care system. There is limited evidence on specific management of postpartum hypertension.

Purpose: Identify risk factors in our community and reduce postpartum readmissions for hypertension within our hospital.

Methods: We performed a retrospective chart review from November 2014 to November 2015. We collected demographic data, comorbidities and information regarding hospitalization and readmission. In this, we identified 28 readmissions for postpartum hypertension, representing $57 \%$ of obstetric readmissions and noted that discharge instructions and blood pressure monitoring postpartum were two areas for improvement. Only $18 \%$ had printed instructions regarding postpartum hypertension. Via multidisciplinary education sessions, we aimed to increased surveillance for postpartum vitals for at-risk patients and provide appropriate verbal and written precautions for signs and symptoms of de novo or worsening hypertensive disease. We also improved access to care by scheduling blood pressure checks within 72 hours of discharge and utilization of visiting nursing services for blood pressure checks. The same measures were then recollected for readmissions from June 2016 to December 2016.

Results: After intervention, $61 \%$ of readmissions were related to hypertension, with 31 readmissions. Overall, there was a significant improvement in written discharge instructions regarding postpartum hypertension, with $94 \%$ receiving written instructions. At discharge, $33 \%$ had blood pressure checks and 13\% had visiting nursing services arranged. 
Conclusion: Postpartum hypertension is more recognized, and readmissions are becoming more common. We increased efforts to optimize medical management of hypertension and reduce preventable readmissions. Improvement in discharge instructions for patients did not decrease overall admission for postpartum hypertension but may have improved overall patient care. Overall cost analysis would be beneficial to see further economic impact.

\section{Robustness of a Newly Proposed Risk Schema for Lymphatic Dissemination in Endometrioid Endometrial Cancer}

Danielle M. Greer, Jessica J.F. Kram, Callie M. Cox Bauer, Scott A. Kamelle

Aurora University of Wisconsin Medical Group; Center for Urban Population Health; Department of Obstetrics and Gynecology, Brook Army Medical Center; Aurora Gynecologic Oncology

Background: Surgical management for endometrioid endometrial cancer (EEC) includes complete lymph node dissection for all patients at risk of lymphatic dissemination. The standard risk schema, defined by Mayo Clinic, identifies low-risk patients as those with grade $1 / 2$ EEC, myometrial invasion $(\mathrm{MI}) \leq 50 \%$, and tumor diameter (TD) $\leq 2 \mathrm{~cm}$. We recently proposed (and published) a risk schema containing modified forms of grade, MI and TD that suggests a significant decrease in false-negative rate and need for lymphadenectomy in low-risk women.

Purpose: Evaluate robustness of our proposed schema for lymphatic dissemination risk stratification in a subsequent EEC patient cohort. Methods: We retrospectively applied the proposed schema to patients diagnosed with stage I-III EEC during 2014-2015 who underwent pelvic and/or para-aortic lymph node removal. Cancer Registry data were confirmed via chart review. Consistent with the cohort studied during model development, the validation cohort included non-Hispanic white or black patients with complete data describing TD ( $\leq 50 \mathrm{~mm}$ or $>50 \mathrm{~mm})$, MI $(\leq 33 \%,>33 \%$ to $\leq 66 \%$, or $>66 \%$ ) and grade (1 or $2-3)$.

Results: In the validation cohort, 29 (11.7\%) of the 247 EEC patients were node-positive (vs $9.2 \%$ of 737 patients in the development cohort). Risk stratification using the proposed schema produced similar false-positive rates during model development (57.2\%) and validation $(54.6 \%)$, both $20 \%$ lower than when using the standard schema $(76.2 \%$ and $74.3 \%$, respectively). False-negative rates, however, were noticeably different between development and validation cohorts using both the proposed $(0 \%$ and $13.8 \%)$ and standard $(1.47 \%$ and $6.90 \%)$ schemas, suggesting a shift toward lowrisk classification in node-positive patients of the validation cohort. Conclusion: Application of the proposed risk stratification schema to an alternative patient cohort verified the utility of modified risk criteria, including TD with 50-mm cutoff, for identifying low-risk EEC patients who may not require node evaluation. However, in the validation cohort, greater prevalence of lymph node metastasis and low-risk classification of node-positive patients was observed. Discrepancy between cohorts is likely due to greater utilization of sentinel lymph node mapping during the validation period, allowing for increased detection of low-volume metastases. Continued model development and validation is needed, especially to account for the increased sensitivity of new technologies.
FMT Placed by Colonoscopy: Systematic Review and Meta-Analysis

Nadia Huq, Veena Kumaravel, Aboud Affi, Maharaj Singh

Departments of Internal Medicine and Gastroenterology, Aurora Sinai Medical Center; Aurora Research Institute

Background: Fecal transplants are successful in the treatment of recurrent or refractory Clostridium difficile infections (CDI), but there is no consensus on the best method of instillation. Studies have shown greater success with lower gastrointestinal tract placement, but technical aspects of placement are not validated.

Purpose: This review aims to identify common traits and procedural techniques of successful fecal microbiota transplant (FMT) therapy via colonoscopy.

Methods: An electronic search was conducted using OVID Medline and PubMed for articles published from January 2010 to January 2016. The primary outcome of interest was cure by FMT placed via colonoscopy.

Results: Of the 337 articles reviewed, we included 24 studies, from which 11 case reports were excluded from data analysis. The resultant data included 366 patients (64\% female). Point estimate for cure of CDI after FMT for patients over 65 years of age $(9 \%)$ was $84.6 \%$ (95\% confidence interval [CI]: $0.58-0.96 ; \mathrm{P}<0.016)$, cure over the age of 18 with no upper limit specified on age $(74 \%)$ was $85.4 \%$ (95\% CI: $0.76-0.91 ; \mathrm{P}<0.001)$ and, for those identified strictly as $18-65$ years old (17\%), cure was $93 \%$ (95\% CI: $0.83-$ $0.98 ; \mathrm{P}<0.001)$. Patients who stopped antibiotics at least 48 hours prior to FMT (37\%) had a cure rate of $86 \%$ (95\% CI: $0.78-0.91$; $\mathrm{P}<0.001)$ compared to $95 \%(95 \% \mathrm{CI}: 0.90-0.98 ; \mathrm{P}<0.001)$ in patients who stopped antibiotics at least 24 hours prior to FMT $(43 \%)$ and $81 \%(95 \%$ CI: $0.53-0.94 ; \mathrm{P}<0.035)$ in those who stopped less than 24 hours prior to FMT (15\%). In studies that specified use of GoLYTELY ${ }^{\circledR}$ prep prior to colonoscopy (58\%), cure was $91 \%$ (95\% CI: 0.85-0.95; $\mathrm{P}<0.001$ ); whereas those using a split $2-\mathrm{L}$ polyethylene glycol prep (21\%) had $79 \%$ cure $(95 \%$ CI: $0.61-0.90$; $\mathrm{P}<0.004)$. Placement of FMT throughout the colon $(6.8 \%)$ had $96 \%$ cure (95\% CI: $0.77-0.99 ; \mathrm{P}<0.002)$ versus terminal ileum to cecum placement $(59 \%)$ at $88 \%$ cure $(95 \% \mathrm{CI}: 0.78-0.94 ; \mathrm{P}<0.001)$ and cecum to ascending colon $(28 \%)$ at $86 \%$ cure (95\% CI: $0.63-0.95$; $\mathrm{P}<0.006)$. Studies that specified the use of loperamide after FMT $(21 \%)$ had a cure of $85 \%$ (95\% CI: $0.63-0.95$; $\mathrm{P}<0.004)$.

Conclusion: FMT placed by colonoscopy has a role in the cure of recurrent or refractory CDI. Stopping antibiotics 24 hours prior to FMT results in higher percentage cure (95\%). Distribution of FMT throughout the colon has better outcomes than FMT instillation at other locations. Effect of loperamide post-FMT placement is not conclusive due to the low percentage of reported use. Prospective studies are recommended to study these factors for confirmation of effects.

\section{Six-Year Experience of Influenza Vaccination as a Condition of Employment for a Large Regional Health Care System}

John R. Brill, Mark Hermanoff, Angela Tonozzi, Mary Jo Capodice, Jennifer Farrar, Zarina Dawoodbhai

Departments of Population Health, Immunology, Infection Control, Occupational Health, Employee Health, and Practice Optimization, Aurora Health Care 